\title{
Field-dependent surface resistance for superconducting niobium accelerating cavities
}

\author{
Wolfgang Weingarten* \\ CERN-BE, CH-1211 Geneva 23, Switzerland \\ (Received 11 April 2011; published 20 October 2011)
}

\begin{abstract}
Superconducting cavities made from niobium allow accelerating gradients of about $50 \mathrm{MV} / \mathrm{m}$ close to the theoretical limit. Quite often, however, the rf losses increase with the gradient faster than quadratic. This observation is equivalent with a decrease of the quality factor $Q$ with the gradient, called " $Q$ slope" for intermediate gradients, and " $Q$ drop" for larger ones. The paper provides an explanation by an elementary model based on the two-fluid theory of rf superconductivity and applies it to experimental data for a large variety of cavity tests.
\end{abstract}

DOI: 10.1103/PhysRevSTAB.14.101002

\section{INTRODUCTION}

The performance of superconducting accelerating cavities improved quite significantly during the past decade and could be pushed up to the theoretical limits of the accelerating gradient for individual cavities. Nevertheless, performance limitations remain and are responsible for a relatively large scatter of performance, even if an identical cavity preparation protocol is closely followed. They are of stochastic nature (e.g. field emission, quenches, residual rf losses, etc.) or of deterministic nature. The latter present hard limits for the prominently applied technology of superconducting cavities made from bulk niobium. These hard limits concern the maximum accelerating gradient (about $50 \mathrm{MV} / \mathrm{m}$ ) and the rf losses associated. They are determined by theoretical foundations, mostly well understood, related to the theory of superconductivity. These are the so-called superheating critical magnetic field, closely related to the thermodynamic critical magnetic field, for the gradient, and the temperature dependent surface resistance for the rf losses. The surface resistance is described by the BCS theory and does not depend on the gradient (except for very high gradients). However, in reality it increases with the gradient, thus imposing additional rf losses to all kinds of superconducting cavities studied so far. These experimental findings lack explanation. It is the purpose of this paper to contribute to the understanding of the increase of the surface resistance with the gradient.

If the tangential $\mathrm{rf}$ electric field $E$ at the cavity surface (which is very small) follows the $\mathrm{rf}$ magnetic field $H=$ $B / \mu_{0}$ in a linear relation, their ratio $E / H$, i.e., the surface resistance $R_{s}$, and hence the $Q$ value $Q \sim 1 / R_{s}$, is constant with $B$. If, however, a nonlinear relation exists between $E$ and $H$, the surface resistance $R_{s}$ decreases or increases

\footnotetext{
*wolfgang.weingarten@cern.ch
}

Published by the American Physical Society under the terms of the Creative Commons Attribution 3.0 License. Further distribution of this work must maintain attribution to the author(s) and the published article's title, journal citation, and DOI.
PACS numbers: 74.25.nn, 74.45.+c, 74.70.-b, 64.60.ah

with $B$. Therefore in this paper the according contribution to the total surface resistance is called "field dependent," $R_{s, f d}$.

In fact three different regimes are observed in niobium accelerating cavities, where the $Q$ value depends on $B$. In the low field region $(B<20 \mathrm{mT})$ the $Q$ value is observed to increase with $B$ (low field $Q$ increase). In the intermediate field region (20-120 mT), the $Q$ value decreases, and beyond, incidentally, the $Q$ value may drop even faster. These latter two observations are named " $Q$ slope" and " $Q$ drop."

The paper is organized as such: In the first section, some general remarks concerning the fitting procedure justify the approach chosen. In the second section the different contributions to the surface resistance are derived from first principles. In the third section, the results of the data analysis are commented in reference to the different contributions and fit parameters as shown in the Appendix, where the chi-square minimization plots are presented. In the fourth section the model is exposed to other experimental findings on niobium cavities. A conclusion summarizes the main results of the paper.

\section{DATA EXTRACTION AND FITTING}

\section{A. Determination of the surface resistance from the $Q$ value}

Whereas the surface resistance $R_{S}$ cannot be measured directly, the $Q$ value can. It is defined as

$$
Q=\omega \frac{\mu_{0} \int d v B^{2}}{\int d a R_{s} B^{2}}
$$

$\omega=2 \pi f$ being the $\mathrm{rf}$ frequency. The integral in the numerator is taken over the volume of the cavity and the integral in the denominator is taken over the surface of the cavity, $B$ being the local magnetic field in the volume or on the surface, respectively. If the surface resistance was constant with $B$, it is inversely proportional to the $Q$ value, 


$$
R_{s}=\frac{G}{Q}
$$

$G$ being the geometry factor

$$
G=\omega \frac{\mu_{0} \int d v B^{2}}{\int d a B^{2}}
$$

But this is in the strict sense only approximately valid for real cavities.

The measurement provides the $Q$ value as a function of the magnetic field $B$. If $B$ is identified with the peak magnetic surface field $B_{p}$, the losses are obviously underestimated and must be corrected.

Normalizing $B$ to $B_{p}$, Eq. (1) transforms into

$$
Q\left(B_{p}\right)=\omega \frac{\mu_{0} \int d v\left(B / B_{p}\right)^{2}}{\int d a R_{s}(B)\left(B / B_{p}\right)^{2}} .
$$

The average surface resistance $\left\langle R_{s}\right\rangle$ is defined as

$$
\left\langle R_{s}\right\rangle\left(B_{p}\right)=\frac{G}{Q\left(B_{p}\right)} .
$$

The local surface resistance $R_{S}(B)$ may be written as a series expansion

$$
R_{s}(B)=R_{s 0}+R_{s}^{\prime} B^{2}+R_{s}^{\prime \prime} B^{4}+\cdots,
$$

which is justified later. A linear dependence on $B$ of the surface resistance $R_{s}(B)$ is not discussed here, because the data fitting does not require it, in accordance with results published elsewhere [1]. From Eqs. (4)-(6), the average surface resistance reads then

$$
\begin{gathered}
\left\langle R_{s}\right\rangle\left(B_{p}\right)=R_{s 0}+R_{s}^{\prime} \underbrace{\frac{\int d a B^{4}}{B_{p}^{2} \int d a B^{2}}}_{\beta^{\prime}} B_{p}^{2}+R_{s}^{\prime \prime} \\
+\cdots .
\end{gathered}
$$

This expression is abbreviated as

$$
\left\langle R_{s}\right\rangle\left(B_{p}\right)=R_{s 0}+\alpha^{\prime} B_{p}^{2}+a^{\prime \prime} B_{p}^{4}+\cdots .
$$

The directly measured coefficients $\alpha^{\prime}, \alpha^{\prime \prime}, \ldots$ of the series expansion of the average surface resistance $\left\langle R_{s}\right\rangle$ are linked with the required coefficients $R_{s}{ }^{\prime}, R_{s}{ }^{\prime \prime}, \ldots$ of the series expansion of the local surface resistance $R_{s}$ as

$$
R_{s}^{\prime}=\frac{\alpha^{\prime}}{\beta^{\prime}}, \quad R_{s}^{\prime \prime}=\frac{\alpha^{\prime \prime}}{\beta^{\prime \prime}} .
$$

The coefficients $\beta^{\prime}, \beta^{\prime \prime}, \ldots$ were computed with SUPERFISH [2] and are summarized here for an elliptically shaped accelerating cavity with a cell length adapted to particles with a velocity close to the velocity of light: $\beta^{\prime}=0.95, \beta^{\prime \prime}=0.92, \beta^{\prime \prime \prime}=0.90, \ldots$

It turns out from the detailed analysis that the average surface resistance $\left\langle R_{s}\right\rangle$ [Eq. (5)] underestimates the local surface resistance $R_{s}\left(B_{p}\right)$ [Eq. (6)] by less than $10 \%$, as long as the ratio $B / B_{c}<0.9$ ( $B_{c}$ is the thermodynamic critical magnetic field). This deviation is considered to be tolerable, because it lies in the same range as the normal measurement error for the $Q$ value. However, for cavity shapes different from the elliptical shape, the deviation is much larger $(\sim 50 \%)$. Therefore the data analysis as presented here is applied exclusively for elliptically shaped cavities.

The surface resistance characterizing the individual loss mechanisms is additive, provided that the rf losses are smoothly distributed over the cavity surface. This fact can be seen from the following argument.

The average surface resistance $R_{s}$ as defined in Eq. (5) can be written as

$$
\frac{1}{Q\left(B_{p}\right)}=\frac{\left\langle R_{s}\right\rangle\left(B_{p}\right)}{G} .
$$

On the other hand, with $P$ being the dissipated power and $U$ the stored energy, $1 / Q$ is defined as

$$
\frac{1}{Q\left(B_{p}\right)}=\frac{P}{\omega U} \text {. }
$$

The different loss contributions $1,2, \ldots$ to the dissipated power $P$ are additive, such that

$$
P=P_{1}+P_{2}+\cdots,
$$

provided that the different losses do not depend on the location on the surface but only on the magnetic field $B$. Then a corresponding individual surface resistance $R_{s 1}, R_{s 2}, \ldots$ may be defined, such that

$$
\begin{aligned}
\frac{1}{Q\left(B_{p}\right)} & =\frac{P_{1}+P_{2}+\cdots}{\omega U} \\
& =\frac{\frac{1}{2} \int d a\left(R_{s 1} B^{2}+R_{s 2} B^{2}+\cdots\right)}{\frac{\omega \mu_{0}}{2} \int d v B^{2}} \\
& =\left(R_{s 1}+R_{s 2}+\cdots\right) \underbrace{\frac{\int d a}{\omega \mu_{0} \int d v}}_{=1 / G} \\
& =\frac{R_{s 1}+R_{s 2}+\cdots}{G} .
\end{aligned}
$$

Hence, the different contributions to the surface resistance are additive. If, however, the rf losses are concentrated in specific regions, the average surface resistance $\left\langle R_{s}\right\rangle$, as defined in Eq. (5), represents a lower limit to the local surface resistance $R_{s}$.

\section{B. Description of the fitting procedure}

The data consist of about 1300 quadruples $\left(R_{s}, B, f, T\right)$ collected from cavity tests of a very broad provenience with regard to the surface resistance $R_{s}$ (tacitly taken 
as $\left\langle R_{s}\right\rangle$ ), magnetic field amplitude $B$ (tacitly taken as $B_{p}$ ), temperature $T$, frequency $f$, shape, cell number, surface treatment, niobium quality, etc. [3-29]. The data are called "collective" sample. The chosen approach aims to cancel out stochastic factors and let prevail the fundamental parameters of the niobium metal.

A relatively large standard deviation of $\sigma=0.35$ of the individual data for $R_{s}{ }^{i}$ was chosen to take into account not only the measurement errors but also variations in preparation and test conditions, cavity types, etc. This led to a minimum chi square for the best guess of the fit parameters $a_{1}, a_{2}, \ldots$ of

$$
\begin{aligned}
\chi^{2}\left(a_{1}, a_{2}, \ldots\right) & =\sum_{i=1}^{n}\left(\frac{R_{s}^{i}-R_{s}\left(B_{i}, f_{i}, T_{i}, a_{1}, a_{2}, \ldots\right)}{\sigma R_{s}^{i}}\right)^{2} \\
& \approx 1200 .
\end{aligned}
$$

This number is reasonably compatible with the total number of about $n \approx 1300$ quadruple individual data.

Several precautions were taken for the fit. For instance, the relevant temperature $T$ is not the helium bath temperature but that of the cavity interior surface. $T$ is determined from the power flux, depending on the measured values of $B$ and $R_{s}$, by taking into account the heat transport properties from inside the cavity to the outside helium bath. The heat transfer depends on the niobium-helium interface thermal boundary resistance, the thermal conductivity, which is related to the residual resistivity ratio $R R R$ of niobium, and the cavity wall thickness. The thermal boundary resistance between niobium and helium- $\mathrm{I}$ is taken into account by data on the nucleate boiling heat transfer [30], up until the film-boiling limit and beyond. The thermal boundary resistance between niobium and helium-II is described by data on the Kapitsa resistance [31]. The heat conductivity of niobium is set proportional to the residual resistivity ratio $R R R$, normalized to $R R R=$ 100 [32]. However, a variation of the heat transport parameters by a factor of 3 upwards or downwards from the $\chi^{2}$ minimum leads to a variation of the fit parameters far below their error and are therefore not considered as relevant.

The total surface resistance of the collective data was fitted with the Mathematica ${ }^{\circledR}$ software by trial functions based on best physical guess. After iterations, the one resulting in the smallest chi square was retained.

The approach was therefore an inductive one, based on the set of experimental collective data and existing knowledge. For reasons of clarity, however, a deductive approach will be chosen in what follows. As a starting point, the known contributions to the surface resistance, expressed as equations with well-defined parameters, are considered. The number of unknown fit parameters is thus reduced to the strict minimum.

\section{QUANTITATIVE ANALYSIS OF THE SURFACE RESISTANCE}

\section{A. Two-fluid model description of the surface resistance}

The electric current in the two-fluid model description for superconductivity [33] is transported by two fluids of charge carriers, the normal conducting and the superconducting fluid. They are considered as noninteracting. The normal-conducting component has a finite resistance or conductance, the superconducting component has inductance but no resistance. The voltage induced across the inductance acts on the resistance and produces $\mathrm{rf}$ losses. From the law of induction follows for the surface resistance $R_{s}$ :

$$
R_{s}=\mu_{0}^{2} \sigma_{n}(T) \omega^{2} \Delta x^{3}
$$

with the rf frequency $\omega=2 \pi f$, the conductivity $\sigma_{n}(T)$ of the normal-conducting fluid, and the penetration depth $\Delta x$ of current flow. In the London model for rf superconductivity $\Delta x$ is the London penetration depth $\lambda_{L}$. The conductivity $\sigma_{n}(T)$ is proportional to the density of normal-conducting electrons and depends on the temperature $T$ as

$$
\sigma_{n}(T)=\sigma_{n 0}\left(\frac{T}{T_{c}}\right)^{4}, \quad \sigma_{n 0}=R R R \sigma,
$$

$\sigma_{n 0}$ being the normal state conductivity at $4.5 \mathrm{~K}, R R R$ the residual resistivity ratio, $\sigma$ the room temperature conductivity, and $T_{c}$ the critical temperature.

The surface resistance $R_{s}$ depends not only on the experimentally controllable variables, such as $B, f, T$, but also on the "parameters," such as $\sigma, R R R, \lambda$, the critical temperature $T_{c}$, etc. Some of them are well known, such as $\sigma, T_{c}$, etc., and are therefore not subject to the fitting. Others are determined by the fit, such as $R R R$, etc. The fit results are then checked on plausibility.

The total surface resistance $R_{s}$ is composed of a sum of the following contributions: (i) the "BCS" surface resistance $R_{s, \mathrm{BCS}}(f, T)$; (ii) the field-dependent surface resistances, $R_{s, f d}(B, f, T)$, describing the $Q$ slope and the $Q$ drop, and $R_{s, Q \text {-inc }}(B, f, T)$, describing the low field $Q$ increase; (iii) the residual surface resistance $R_{s, \text { res }}$, and possibly others.

\section{B. The BCS surface resistance}

Instead of solving the Mattis-Bardeen integrals [34] for the surface resistance, the following analysis is based, for reasons of clarity, on the two-fluid model. The temperature dependence of the surface resistance $R_{S}$ as described by Eq. (16), is modified following the BCS theory [35]:

$$
R_{s, \mathrm{BCS}}(\omega, T)=\mu_{0}^{2} \omega^{2} \sigma_{n 0} \lambda^{3} \frac{\Delta}{k_{B}} \ln \left(\frac{\Delta}{\hbar \omega}\right) \frac{e^{-\Delta / k_{B} T}}{T},
$$

$\Delta$ being the superconducting energy gap and $k_{B}$ being the Boltzmann constant. The surface resistance of Eq. (17) 
depends besides the temperature $T$ and the frequency $\omega$ also on the mean-free path $l$, as indicated here:

$$
\lambda(T, l)=\frac{\lambda_{0}(l)}{\sqrt{1-\left(\frac{T}{T_{c}}\right)^{4}}} ; \quad \lambda_{0}(l)=\lambda_{L} \sqrt{1+\frac{\xi_{0}}{l}},
$$

with $\lambda_{0}$ the penetration depth at $T \rightarrow 0 \mathrm{~K}$, and $\lambda_{L}$ the London penetration depth. The coherence length $\xi$, to be used later, is

$$
\xi(l)=\frac{1}{\frac{1}{\xi_{0}}+\frac{1}{l}},
$$

$\xi_{0}$ being the coherence length for pure niobium. The Ginzburg-Landau parameter $\kappa$ is the ratio of penetration depth $\lambda$ and coherence length $\xi: \kappa=\lambda / \xi$. The conductivity $\sigma_{n 0}$ of the normal-conducting electrons depends on the mean-free path $l$, too,

$$
\sigma_{n 0}=\frac{l e^{2}}{m v_{F}} n_{n 0}
$$

with the electron mass $m$, the Fermi velocity $v_{F}$, and the density just above $T_{c}$ of the normal-conducting electrons $n_{n 0}$. Equation (20) allows the replacement of the dependence on the mean-free path $l$ by a dependence on the residual resistivity ratio $R R R$,

$$
l l_{\mathrm{nm}}=2.7 R R R \text {. }
$$

In reality, the density of normal-conducting electrons may be increased by the presence of normal-conducting defects, located to a depth $\Delta y$ away from the surface, but within the penetration depth $\lambda$ of the electromagnetic field. These surplus normal-conducting electrons contribute to the rf dissipation in the same way as those from the normalconducting two-fluid model component. Therefore their share of $\mathrm{rf}$ dissipation is taken into account by a frequency dependent residual surface resistance $R_{\text {res }}(\omega)$. It should have the same frequency dependence as the BCS surface resistance, because it is created by the same physical effect. Using the fundamental equation for the two-fluid model surface resistance, Eq. (15), the frequency dependent residual surface resistance is

$$
R_{\text {res }}(\omega)=\mu_{0}^{2} \sigma_{n 0} \omega^{2} \lambda^{2} \Delta y .
$$

The contribution $R_{\text {res }}$ accounts for experimental results as published elsewhere [36,37].

\section{The field-dependent surface resistance}

The field-dependent surface resistance comprises the contribution $R_{s, f d}$ for the $Q$ slope and $Q$ drop and the contribution $R_{s, Q \text {-inc }}$ for the low field $Q$ increase. The field-dependent surface resistance $R_{s, f d}$ factorizes in a good approximation into a temperature dependent and a field-dependent part, $R_{s, f d}(B, \omega, T) \approx R_{s, \text { fdt }}(\omega, T)$.
$R_{s, f d b}(B)$, as will be shown later. This observation was also noticed for niobium film cavities [38,39].

\section{The field dependence of $\boldsymbol{R}_{s, f d}$}

\section{a. The interface vacuum-superconductor with defect}

The superconducting surface of niobium is supposed to be imperfect in a sense as to allow the entry of magnetic flux (no Bean-Livingston barrier). It may, for instance, contain a normal-conducting "defect," acting as a "condensation nucleus," with radius $a$ small compared to the characteristic length scales in a superconductor (coherence length $\xi$ and penetration depth $\lambda$ ). The origin of such a defect is explained later. The important notion to be retained lies in the fact that a planar geometry is replaced by a spherical geometry.

We suppose that the surface with defect is exposed to an rf magnetic field $B$. Inspecting Fig. 1, at the interface between the normal-conducting defect and the circumjacent superconductor, being of type II, the Cooper pair density increases relatively rapidly away from the defect on a characteristic length scale $\xi$, defining the condensation volume $V_{c}$. Much further away, the shielding action against $B$ by the Meissner-Ochsenfeld effect becomes effective on a length scale of typically $\lambda>\xi$, defining the magnetic volume $V_{m}$. Hence, compared to a situation where the transition is abrupt, the superconductor provides less condensation energy $\Delta E_{c}$, independent of $B$, and gains more diamagnetic energy $\Delta E_{B}$, proportional to $B$, up to a finite $B^{*}$, where the energy balance is equalized:

$$
\begin{aligned}
\Delta E & =\Delta E_{c}-\Delta E_{B}=\frac{1}{2 \mu_{0}} B_{c}^{2} V_{c}-\frac{1}{2 \mu_{0}} B^{* 2} V_{m}=0 \\
& \Rightarrow B^{* 2} V_{m}=B_{c}^{2} V_{c} .
\end{aligned}
$$

Hence, for

$$
B>B^{*}=\sqrt{\frac{V_{c}}{V_{m}}} B_{c},
$$

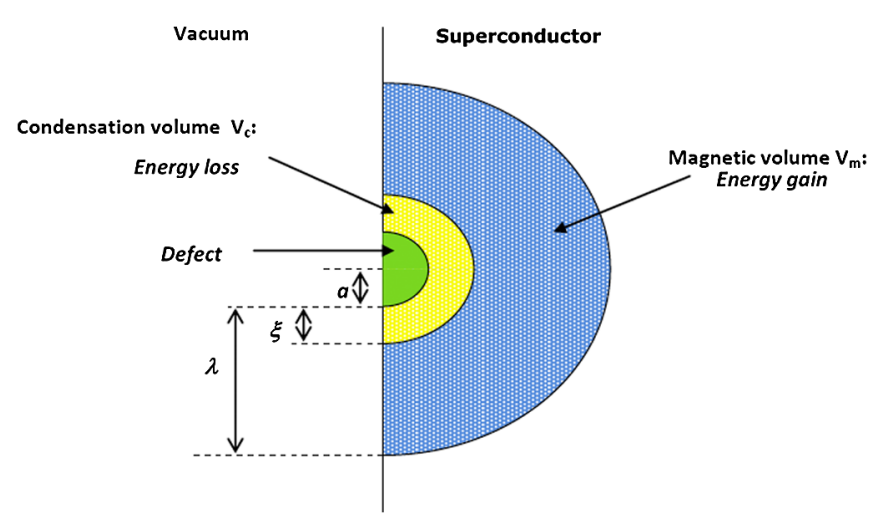

FIG. 1. The superconductor loses energy inside the condensation volume $V_{c}$ and gains energy inside the magnetic volume $V_{m}$. 
the entry of magnetic flux is energetically possible, because this lowers the total energy $E$.

For the special case of an interface between a normalconducting half sphere of radius $a$ embedded at the surface of a superconducting metal, the volumes concerned are

$$
V_{m}=\frac{2}{3} \pi(a+\lambda)^{3} ; \quad V_{c}=\frac{2}{3} \pi(a+\xi)^{3},
$$

from which

$$
\begin{aligned}
& \frac{V_{m}}{V_{c}} \approx 1 \text { for } a \gg \lambda, \xi, \\
& \frac{V_{m}}{V_{c}} \approx\left(\frac{\lambda}{\xi}\right)^{3}=\kappa^{3} \text { for } a \ll \lambda, \xi,
\end{aligned}
$$

as well as

$$
\begin{aligned}
& B^{*} \approx B_{c} \text { for } a \gg \lambda, \xi \\
& B^{*} \approx\left(\frac{\xi}{\lambda}\right)^{3 / 2} B_{c}=\frac{1}{\kappa^{3 / 2}} B_{c} \quad \text { for } a \ll \lambda, \xi
\end{aligned}
$$

$B^{*}$ may therefore become pretty small for dirty superconductors with a small mean-free path $l$ and consequently small coherence length $\xi$. This is one possibility of creating a normal-conducting defect already at very small magnetic fields. But there are others.

Equation (24) may be interpreted as a functional relation between the applied magnetic field $B=B^{*}$ and the associated condensation volume $V_{c}(B)$, with $B_{c}$ and $V_{m}$ being considered as constant. This is obviously valid for magnetic fields small as compared to the critical magnetic field $B_{c}$ and for temperatures small as compared to the critical temperature $T_{c}$. The larger $B$, the larger becomes $V_{c}$ in order to establish the energy balance:

$$
B^{2} V_{m}=B_{c}^{2} V_{c} .
$$

Differentiating Eq. (28) results, for $B \ll B_{c}$, in an expression for the increase of the condensation volume $\Delta V_{c}$ $(B)$ under the incremental increase $\Delta B$ of the applied magnetic field $B$,

$$
\Delta V_{c}(B)=\frac{2 B V_{m}}{B_{c}^{2}} \Delta B
$$

If $B$ approaches $B_{c}$, the magnetic volume $V_{m}$ is no longer constant, and Eq. (29) is modified to

$$
\Delta V_{c}(B)=\frac{2 B V_{m}}{B_{c}^{2}} \Delta B+\left(\frac{B}{B_{c}}\right)^{2} \Delta V_{m} .
$$

In this case, using Eqs. (25) and (30), the following relations hold:

$$
\frac{\Delta V_{m}}{\Delta V_{c}}=\left(\frac{a+\lambda}{a+\xi}\right)^{2} \approx\left(\frac{\lambda}{\xi}\right)^{2}=\kappa^{2} \text { for } a \ll \lambda, \xi
$$

$$
\Delta V_{c}=\frac{2 B V_{m}}{B_{c}^{2}-(\kappa B)^{2}} \Delta B
$$

A distinction must be made between the situation when the defect is located at the surface, and when it is located in the bulk, but still within a distance given by the penetration depth $\lambda$ and, hence, exposed to the rf current.

If the defect is embedded in the bulk, the current passes around it on both sides when becoming normal conducting. In other words, a looplike microscopic magnetic field is created with the net result of zero change of magnetic induction in the superconductor: the diamagnetic energy remains unchanged, the energy balance $\Delta E$ will not become negative, and, hence, no transition from the superconducting state to the normal-conducting state will occur. This is the reason why the normal-conducting volume $V_{c}$ will only grow under the influence of the magnetic field $B$ when located at the surface and not inside the superconductor.

A precondition for growth is therefore the existence of a normal-conducting defect at the surface. It is well known that such defects exist even at zero magnetic field [40].

\section{b. Derivation of the field-dependent surface resistance}

The $Q$ slope and $Q$ drop.-The supposition is made that a relative increase of the condensation volume $\Delta V_{c}$ is accompanied by a relative increase of the electron density $\Delta n_{n}$, which may depend on the temperature $T$,

$$
\frac{\Delta n_{n}}{n_{n}}=\frac{\Delta V_{c}}{V_{c}} .
$$

Differentiating Eq. (20) and using Eqs. (32) and (33), we obtain

$$
\begin{aligned}
\Delta \sigma_{n} & =\frac{l e^{2}}{m v_{F}} \Delta n_{n}=\underbrace{\frac{l e^{2}}{m v_{F}} n_{n}}_{\sigma_{n}} \frac{\Delta V_{c}}{V_{c}} \\
& =\sigma_{n} \frac{V_{m}}{V_{c}} \frac{2 B}{B_{c}^{2}-(\kappa B)^{2}} \Delta B .
\end{aligned}
$$

By the definition of the two-fluid model surface resistance $R_{s}$, Eq. (15), the dissipated power per unit area $p=R_{s}$. $\left(B / \mu_{0}\right)^{2} / 2$ increases with the conductivity as

$$
\Delta p=\frac{1}{2} \omega^{2} \Delta x^{3} B^{2} \Delta \sigma_{n}
$$

$\Delta x$ being of the order of the penetration depth $\lambda$, which is identical with the spatial extension of the magnetic volume $V_{m}$.

Using Eq. (34),

$$
\Delta p=\omega^{2} \Delta x^{3} \sigma_{n} \frac{V_{m}}{V_{c}} \frac{2 B^{3}}{B_{c}^{2}-(\kappa B)^{2}} \Delta B
$$


Equation (36) is integrated from the threshold field $B^{*}$ (taken for convenience as zero, because it is small anyway) to the magnetic field amplitude $B$,

$$
p=-\omega^{2} \Delta x^{3} \sigma_{n} \frac{V_{m}}{V_{c}}\left[\frac{B^{2}}{2 \kappa^{2}}+\frac{B_{c}^{2} \ln \left[1-\kappa^{2}\left(\frac{B}{B_{c}}\right)^{2}\right]}{2 \kappa^{4}}\right] .
$$

By definition of the surface resistance, and taking for convenience $V_{m} / V_{c} \approx 1$ [Eq. (26)] being valid for metals with $\kappa \approx 1$, such as niobium,

$$
\begin{aligned}
R_{s, f d} & =\frac{p}{\frac{1}{2}\left(\frac{B}{\mu_{0}}\right)^{2}} \\
& \approx \underbrace{\mu_{0}^{2} \omega^{2} \Delta x^{3} \sigma_{n}(T)}_{R_{s, f d t}}(-1) \underbrace{\left[\frac{1}{\kappa^{2}}+\frac{\ln \left[1-\kappa^{2}\left(\frac{B}{B_{c}}\right)^{2}\right]}{\kappa^{4}\left(\frac{B}{B_{c}}\right)^{2}}\right]}_{R_{s, f d b}} .
\end{aligned}
$$

Equation (38) shows the factorization of the fielddependent surface resistance $R_{s, f d}$ into a temperature dependent factor $R_{s, f d t}(T)$, via $\sigma_{n}(T)$, and into a fielddependent factor $R_{s, f d b}(B)$ [with a weak temperature dependence, though, via $\left.B_{c}(T)\right]$.

After expansion of the logarithm into an infinite but slowly converging series, Eq. (38) reads

$$
\begin{aligned}
R_{s, f d} & \approx \mu_{0}^{2} \omega^{2} \Delta x^{3} \sigma_{n}(T) \frac{1}{\kappa^{2}}\left(\frac{\left(\frac{\kappa B}{B_{c}(T)}\right)^{2}}{2}+\frac{\left(\frac{\kappa B}{B_{c}(T)}\right)^{4}}{3}+\cdots\right) \\
B_{c}(T) & \approx B_{c, T=0}\left[1-\left(\frac{T}{T_{c}}\right)^{2}\right] .
\end{aligned}
$$

$\kappa$ is the Ginzburg-Landau parameter and $B_{c}$ is the thermodynamic critical field of niobium.

Equations (38) and (39) describe in a common way the $Q$ slope as well as the $Q$ drop, as suggested by the singularity of Eq. (38) at $B=B_{c} / \kappa$.

The low field $Q$ increase.-It is well known that the niobium surface consists of a composite of a niobium matrix that comprises among other elements dissolved gases as interstitials, such as oxygen, various oxides of niobium [40], and hydrogen [41]. As a paradigm, only the case of $\mathrm{NbO}$ is studied in this paper, having in mind, though, that hydrogen may also be of relevance. A composite of $\mathrm{NbO} / \mathrm{Nb}$ on top of, and in close contact with, the niobium bulk will be subject to the superconducting proximity effect [42]. It follows that the $\mathrm{NbO}$ component exhibits a transition temperature $T_{c N S}$ in between the transition temperatures of the two constituents, called " $N$ " for the weak superconductor $(\mathrm{NbO})$ and " $S$ " for the strong superconductor $(\mathrm{Nb})$, whereas the transition temperature of the $\mathrm{Nb}$ is only weakly affected. Not only the critical temperature $T_{c N S}$ of the $N$ component, but also its critical field $B^{*}$ is modified by the presence of the $S$ component. For a sufficiently thick $N$ layer, there is a phase transition of first order, in the presence of a magnetic field, from the superconducting state to the normal-conducting state at very low magnetic field $[42,43]$. This phase transition happens twice per rf cycle and needs a tiny amount of energy, the latent heat $L$. This energy is provided by the rf field that boosts the $N$ component (defect) into the normal-conducting state. As this energy cannot coherently be rendered back to the rf field, it is dissipated as heat. Hence, in the low field region, there exists another contribution to the field-dependent surface resistance that originates from the proximity effect and is proportional to the $\mathrm{rf}$ frequency $\omega=2 \pi f$. As the latent heat $L$ per square meter is independent of the $\mathrm{rf}$ magnetic field amplitude $B$, the dissipated power per square meter is $p=L \cdot 2 f$. The related surface resistance is derived by using the definition $p=R_{s} \cdot\left(B / \mu_{0}\right)^{2} / 2$, as

$$
R_{s, Q \text {-inc }} \approx \frac{2 \omega L \mu_{0}^{2}}{\pi B^{2}}
$$

This equation describes the low field $Q$ increase.

\section{The temperature dependence of $\boldsymbol{R}_{s, f d}$}

a. Proximity effect in the $\mathrm{NbO} / \mathrm{Nb}$ composite:

\section{Determination of $T_{c N S}$}

As outlined before, only the niobium monoxide $(\mathrm{NbO})$ will be considered as a relevant candidate for the proximity effect among the other compounds of the niobium surface composite. It is metallic and a "weak" superconductor with a transition temperature $T_{c}=1.38 \mathrm{~K}$. Other relevant parameters for $\mathrm{NbO}$ are shown in Table I, in comparison with those for $\mathrm{Nb}$. They are the superconducting coupling constant $(N V)$, the Debye temperature $\Theta_{D}$, and the electron density $N$ near the Fermi surface.

The superconducting coupling constants $(N V)$ for $\mathrm{Nb}$ and $\mathrm{NbO}$ are determined from their respective critical temperatures $T_{c}$ via the "BCS formula,"

$$
T_{c}=1.14 \Theta_{D} e^{-(1 / N V)} .
$$

The electron density $N$ for $\mathrm{Nb}$ is calculated from the Fermi velocity $v_{F}$,

$$
v_{F}=\frac{\hbar}{m}\left(3 \pi^{2} N\right)^{1 / 3},
$$

taken from the literature [44]. The electron density $N$ of $\mathrm{NbO}$ is taken from the literature as well [45]. The limiting case is considered here, when the typical spatial extensions of the " $N$ component" and the " $S$ component" are small compared to the coherence length (Cooper limit [46]). The average coupling constant of the composite in the Cooper limit is given by

TABLE I. Superconducting parameters of $\mathrm{Nb}$ and $\mathrm{NbO}$.

\begin{tabular}{lcccc}
\hline \hline & $(N V)_{S, N}$ & $\Theta_{D}[\mathrm{~K}]$ & $T_{c}[\mathrm{~K}]$ & $N_{S, N}\left[\mathrm{~cm}^{-3}\right]$ \\
\hline $\mathrm{Nb}$ & 0.2835 & 276 & 9.25 & $5.56 \times 10^{22}$ \\
$\mathrm{NbO}$ & 0.1677 & 472 & 1.38 & $1.60 \times 10^{22}$ \\
\hline \hline
\end{tabular}




$$
(N V)_{\mathrm{eff}}=\frac{(N V)_{N} N_{N} \boldsymbol{v}_{N}+(N V)_{S} N_{S} \boldsymbol{v}_{S}}{N_{N} \boldsymbol{v}_{N}+N_{S} \boldsymbol{v}_{S}},
$$

$v_{N}$ and $v_{S}$ being the volumes, $N_{N}$ and $N_{S}$ the electron densities, and $(N V)_{N}$ and $(N V)_{S}$ the superconducting coupling constants of the $N$ and $S$ components, respectively.

Once $(N V)_{\text {eff }}$ is known, the critical temperature $T_{c N S}$ is calculated from Eq. (41), taking for the Debye temperature $\Theta_{D}$ the value for $\mathrm{NbO}$, which is the dominant constituent in the composite. Figure 2 depicts the relation between the critical temperature $T_{c N S}$ of the $N$ component vs the volume concentration $x=v_{S} /\left(v_{S}+v_{N}\right)$ of the $S$ component in the $\mathrm{NbO} / \mathrm{Nb}$ composite. The relation of the critical temperature $T_{c N S}$ vs $x$ starts at the critical temperature $T_{c}=1.34 \mathrm{~K}$ for the NbO solely, when the concentration of the $S$ component is zero. The temperature difference $T_{c, N S} T_{c, \text { NbO }}$ follows a quasilinear relation with the concentration $x$,

$$
T_{c N S}-T_{c, \mathrm{NbO}} \sim x .
$$

As a consequence of the proximity effect, the preceding description provides another mechanism by which "defects" are created.

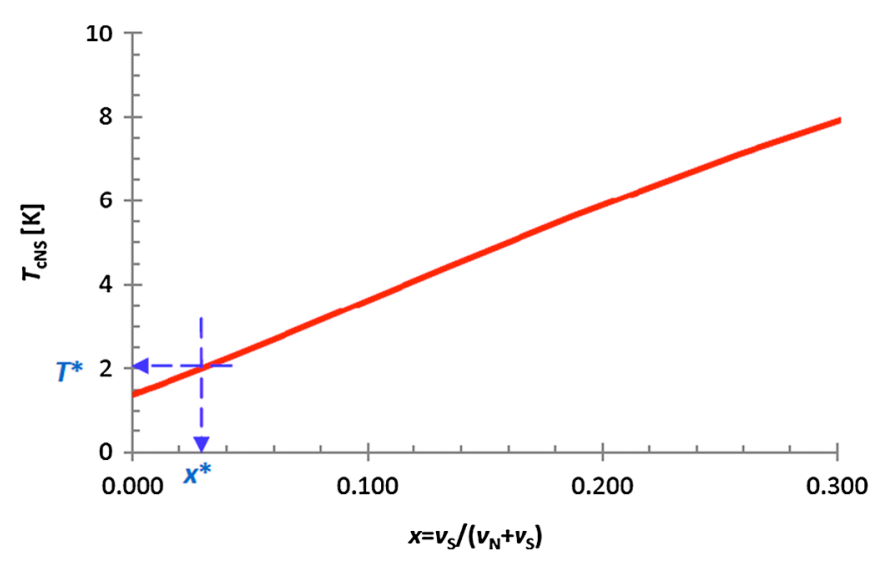

FIG. 2. Critical temperature of the $\mathrm{NbO} / \mathrm{Nb}$ composite in the Cooper limit of the proximity effect vs the volume fraction $x=v_{S} /\left(v_{N}+v_{S}\right)$ of the $S$ component $(\mathrm{Nb})$.

\section{b. Percolation effect in the $\mathrm{NbO} / \mathrm{Nb}$ composite}

Supposing that the helium bath temperature $T$ is increased from the critical temperature $T_{c}=1.38 \mathrm{~K}$ of $\mathrm{NbO}$ further up. Because of the proximity effect, by the presence of $\mathrm{Nb}(S)$, the $\mathrm{NbO}$ is still superconducting. Increasing $T$ further, the $\mathrm{NbO}$ in the composites with the smallest volume fraction of $\mathrm{Nb}$ will first become normal conducting. The $\mathrm{Nb}$ in the composite still remains superconducting, but does not yet form a continuous superconducting path among itself. Increasing the temperature even more, the $\mathrm{NbO}$ in those composites with a larger volume fraction of $\mathrm{Nb}$ will also become normal conducting up to the temperature, where the $\mathrm{Nb}$ in the composite forms a continuous superconducting path among itself. This situation is identical with a so-called percolation threshold (Fig. 3).

It should be noted that, as soon as the $\mathrm{Nb}$ of the composite forms a continuous superconducting path, the $\mathrm{NbO}$ of the composite fragments into normal-conducting regions of small size. The normal-conducting regions provide the small condensation nuclei needed for the entry of magnetic flux, as described before, already at a very small rf magnetic field $B$.

However, as long as the $\mathrm{Nb}$ in the composite does not create a superconducting path, the entire composite itself represents a normal-conducting defect of a size so large that the entry of magnetic flux is prohibited at very small $\mathrm{rf}$ magnetic fields $B$. Obviously, the entire composite contributes to rf losses, as will be discussed later.

Percolation means long-range connectivity in random systems. There is a percolation threshold $x^{*}$ depending on the occupation probability $x$, where infinite connectivity (percolation) first occurs.

It follows as a corollary that long-range connectivity is associated with the generation of isolated sites acting as nucleation centers favoring the entry of magnetic flux.

Percolation thresholds of composites were extensively studied in recent years. For example, the "void percolation threshold" for "continuum percolation," as it is called in the

Nbo embedded in $\mathrm{Nb}$ matrix

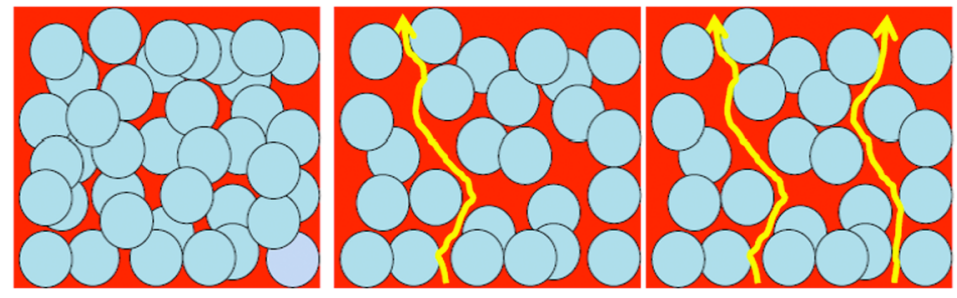

\begin{tabular}{|c|c|c|c|}
\hline $\begin{array}{c}\text { Volume ratio } x \\
\text { of Nb/NbO }\end{array}$ & $x \ll x^{*}$ & $x=x^{*}=0.0301$ & $x » x^{*}$ \\
\hline$T_{\mathrm{cNS}}(\mathrm{NbO})$ & $T_{\mathrm{cNS}} \ll T^{*}$ & $T_{\mathrm{cNS}}=T^{*}=2.015 \mathrm{~K}$ & $T_{\mathrm{cNS}} » T^{*}$ \\
\hline
\end{tabular}

FIG. 3. Schematic of a cluster of equally sized spheres below (left) and above the percolation threshold (middle and right). 
literature, was investigated for a distribution of overlapping spheres $(N)$ with equal radius and voids $(S)$ in between. The threshold volume fraction is $x^{*}=v_{S} /\left(v_{N}+v_{S}\right)=$ $0.0301 \pm 0.0003$ [47]. Above this threshold the voids are connected.

Percolation effects are not only characterized by the percolation threshold concentration $x^{*}$, but also by the critical exponent $\beta$. The probability $P_{\infty}$ that a site belongs to an infinite cluster is zero below $x^{*}$ and increases above $x^{*}$ as

$$
P_{\infty}=\left(x-x^{*}\right)^{\beta}
$$

with the concentration $x$ of "occupied" sites [48]. The critical exponent $\beta$ is universal, i.e., largely independent of the shape of the occupied sites. It depends on the dimensionality of the problem and other features.

The following two examples are relevant for the analysis.

In a finite but large resistor lattice with a random fraction of resistors removed there exists a threshold concentration of resistors $x^{*}$ [49]. For $x<x^{*}$ the lattice is so fragmented that is does not conduct. However, for $x>x^{*}$ there are connected conducting paths of infinite extent and the conductivity obeys a power law $\sigma \sim\left(x-x^{*}\right)^{t}$ with the critical exponent $t=1.1 \pm 0.05$, for "bond" percolation on a twodimensional lattice. Bond percolation means long-range connectivity of the interface (bonds) between cells, precisely what is shown in Fig. 3. Evidence for a dimensionality of 2 was also found for a percolation-driven transition from incoherent to coherent surface superconductivity [50].

\section{c. Conspiracy between percolation and proximity effect}

Applied to the $\mathrm{NbO} / \mathrm{Nb}$ composite, we identify the spheres with the $\mathrm{NbO}$ part of the composite $(N)$, and we identify the voids with the $\mathrm{Nb}$ part of the composite $(S)$. We would therefore expect a continuous path of $\mathrm{Nb}(S)$ to exist, if $x=v_{S} /\left(v_{N}+v_{S}\right)>x^{*}$. Inspecting Fig. 2, this situation corresponds to a transition from superconducting to normal of the $\mathrm{NbO}$ in the composite at $T^{*}=2.015 \mathrm{~K}$, which we call "percolation temperature." Figure 3 illustrates the argument in a different way.

In conclusion, only for temperatures $T>T^{*}$, magnetic flux will enter at relatively small magnetic field amplitudes $B$ and make the condensation volume $V_{c}$ grow under the action of $B$, as described by Eq. (23).

Hence, $R_{s, f d}$ will be relatively small below $T^{*}$.

Replacing $x$ by $T$ in Eq. (45), introducing the percolation temperature $T^{*}$, which is justified due to linear relation between $T_{c N S}-T_{c, \mathrm{NbO}}$ and $x$ [Eq. (44)], and normalizing properly the probability $P_{\infty}$, we obtain

$$
P_{\infty}(T)=\Theta\left(T-T^{*}\right)\left(\frac{T_{c N S}-T^{*}}{T_{c}-T^{*}}\right)^{\beta} .
$$

$\Theta(x)$ is the step function.

For the temperature dependence of the conductivity $\sigma_{n}(T)$ follows therefore

$$
\sigma_{n}(T)=\sigma_{n 0} \Theta\left(T-T^{*}\right)\left(\frac{T-T^{*}}{T_{c}-T^{*}}\right)^{\beta} .
$$

Equation (47) describes how the magnetic field acts on defects thus causing the increase, at the niobium surface, of the conductivity of the normal-conducting electrons above a threshold temperature $T^{*}$. However, the fitting of the collective data imposes considering normal-conducting defects that exist also below $T^{*}$ with a spatial dimension sufficiently small to allow the entry of magnetic flux. It is conjectured that they originate from normal-conducting zones penetrated by the superconducting current (e.g. grain boundaries), thus adding a purely Ohmic contribution to the surface resistance, $R_{\text {res } 1 \text {, independent of the } \mathrm{rf}}$ frequency. Hence, the temperature dependent part of Eq. (38), $R_{s, f d t}$, must be completed by the residual resistance term, $R_{\text {res } 1}$.

\section{Roundup on the field-dependent surface resistance}

In summary, the following conditions are sufficient, in the frame of the proposed model, to allow the entry of magnetic flux $B$ causing the field-dependent surface resistance $R_{s, f d}$ : (i) There exist at the surface tiny isolated "nucleation centers" or "defects," e.g., clusters of $\mathrm{NbO}$, that are normal conducting at zero magnetic field or become normal conducting by the proximity effect in a first order phase transition at very low magnetic fields; the dissipated latent heat is described by the surface resistance as in Eq. (40); (ii) the magnetic flux entry is initiated at these tiny defects and penetrates deeper into the bulk under the action of the magnetic field; (iii) the "conspiracy" of percolation and proximity effects increases the density of normal-conducting electrons starting from a threshold temperature $T^{*}$; these normal-conducting electrons are subject to the inductive voltage as created by the superconducting electrons under the action of the rf field, in a very similar way to the origin of the BCS surface resistance; (iv) the field-dependent surface resistance $R_{s, f d}$ can be described by the formula

$$
R_{s, f d}(\omega, T, B) \approx[R_{\text {res1 }}+\underbrace{\mu_{0}^{2} \omega^{2} \Delta x^{3} \sigma_{n 0} \Theta\left(T-T^{*}\right)\left(\frac{T-T^{*}}{T_{c}-T^{*}}\right)^{\beta}}_{R_{s, f d t}}] \underbrace{\frac{1}{\kappa^{2}}\left(\frac{\left(\frac{\kappa B}{B_{c}(T)}\right)^{2}}{2}+\frac{\left(\frac{\kappa B}{B_{c}(T)}\right)^{4}}{3}+\cdots\right)}_{R_{s, f d b}} .
$$




\section{Other contributions to the surface resistance}

As mentioned before, other however small contributions to the surface resistance must be added. They are imposed when fitting the collective data. It is conjectured that they originate from normal-conducting zones penetrated by the superconducting current (e.g. grain boundaries). The corresponding losses are Ohmic in nature and hence do not depend on the rf frequency. The according surface resistance is named $R_{\text {res } 2}$.

\section{RESULTS OF DATA ANALYSIS}

\section{A. Contributions to the surface resistance}

\section{The BCS surface resistance $R_{s, \mathrm{BCS}}$}

The task of data analysis consists in extracting the fielddependent and other contributions to the surface resistance from the total surface resistance $R_{s}$. This is achieved by subtracting from $R_{s}$ the well-known contributions, such as the BCS surface resistance $R_{s, \mathrm{BCS}}$ and the residual surface

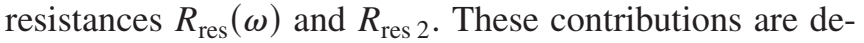
scribed by Eqs. (17) and (22). The fixed parameters for $R_{s, \mathrm{BCS}}$ are the energy gap $\Delta$ and the room temperature conductivity of niobium $\sigma$. The fit parameter is the resistivity ratio $R R R$. The $\mathrm{BCS}$ surface resistance $R_{S, \mathrm{BCS}}$ is determined by adapting the fixed parameters of Eq. (17) in such a way as to closely approximate published data [51]. The $R R R$ value of the niobium cavities on which the published data were taken is not precisely known. A typical number is $R R R=40$, generally valid for reactor-grade niobium commercially available at the time when the data were published. Then Eq. (17) overestimates the data by a factor 4. Nevertheless best fits were obtained when the BCS resistance was lowered by an additional factor of 2.5.

\section{The field-dependent and other contributions to the surface resistance}

\section{a. Fitting the individual data}

The individual data are analyzed as follows. The measured total surface resistance $R_{s}$ is fitted by the formulas as summarized in the Appendix. The fitted contributions to the surface resistance from the BCS and residual parts, as well as from the low field $Q$ increase, are subtracted from the measured total surface resistance $R_{s}$. What remains is dominated by the field-dependent surface resistance $R_{s, f d}$. The observation is used that the factor in Eq. (48), $R_{s, f d b}$, describes pretty well the field-dependent part. What is plotted then in Fig. 4 is the remaining temperature dependent factor $R_{s, f d t}$, after subtraction of the small residual part $R_{\text {res } 1}$, and conveniently averaged and normalized to $400 \mathrm{MHz}$.

All these fits to the individual data indicate that $R_{s, f d t}$ does not depend on the magnetic field $B$, but only on the temperature, increasing steeply above a threshold temperature $T^{*}$ and being small below. The data suggest that the field-dependent surface resistance $R_{s, f d}$ factorizes into a

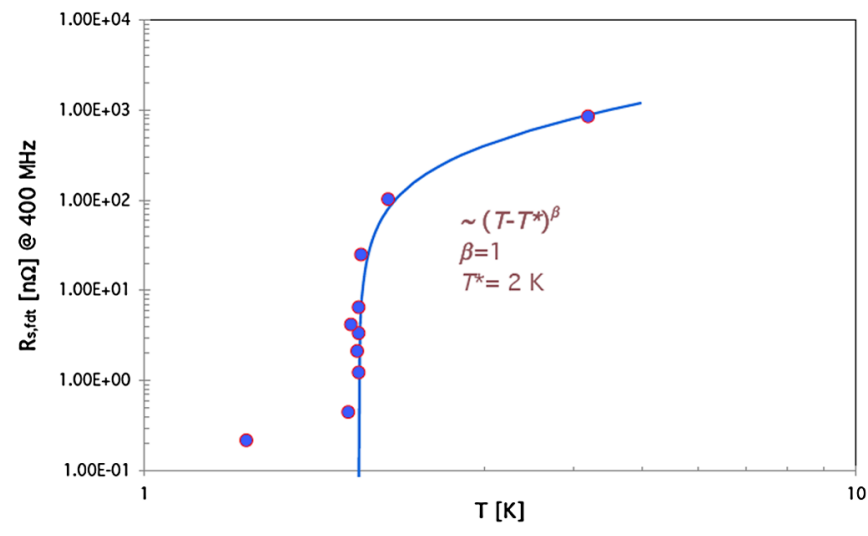

FIG. 4. The averaged temperature dependent part of the fielddependent surface resistance $R_{s, f d t}$ versus the bath temperature $T$ (the error bars are smaller than the data points).

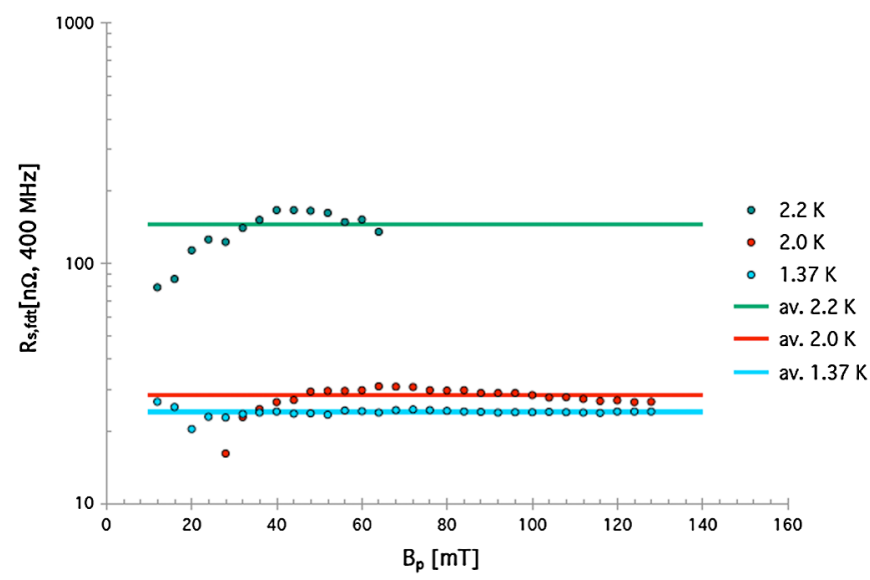

FIG. 5. Data highlighting the nondependence of the temperature dependent part of $R_{s, f d}$ on the magnetic field $B$; the individual data were taken at three different bath temperatures for one cavity.

field-dependent and a temperature-dependent part. This factorization is illustrated in Fig. 5 for a representative individual data set [12].

The straight lines represent, after a removal of outliers, the average values to the individual data that were used for plotting Fig. 4.

\section{b. Fitting the collective data}

Having gained evidence for the factorization of $R_{s, f d}$ from the individual data, the analysis of the collective data is done in a similar way. All fixed original and derived parameters as used for the $\chi^{2}$ minimization are listed in Table II.

All fitted original and derived parameters as used for the $\chi^{2}$ minimization are listed in Table III. The numbers are taken from the $\chi^{2}$ minimization as shown in the Appendix.

\section{B. Discussion}

As to the BCS surface resistance, the fit of the collective data yields $R R R=176$, corresponding to a mean-free path 
TABLE II. Fixed original and derived parameters used by $\chi^{2}$ minimization of the collective data.

\begin{tabular}{lll}
\hline \hline Original parameter & Value & Unit \\
\hline$\Delta / k_{B}$ & 16.2 & $\mathrm{~K}$ \\
$\sigma$ & $7.6 \times 10^{-6}$ & $(\Omega \mathrm{m})^{-1}$ \\
$T^{*}$ & $2.07^{\mathrm{a}}$ & $\mathrm{K}$ \\
$B_{c}$ & 0.190 & $\mathrm{~T}$ \\
$T_{c}$ & 9.25 & $\mathrm{~K}$ \\
$\lambda_{L}$ & 38 & $\mathrm{~nm}$ \\
$\xi_{0}$ & 38 & $\mathrm{~nm}$ \\
$\kappa$ (surface) & 0.8 & $\cdots$ \\
Nb wall thickness & 2.5 & $\mathrm{~mm}$ \\
\hline \hline
\end{tabular}

${ }^{\text {a }}$ This temperature $T^{*}$ denotes the one at the inside of the cavity. It is estimated to $70 \mathrm{mK}$ above the one of Fig. 4, which denotes the helium bath temperature. The difference is caused by the heat transport across the niobium wall to the helium bath.

TABLE III. Fitted original and derived parameters obtained by $\chi^{2}$ minimization of the collective data.

\begin{tabular}{lll}
\hline \hline Original parameter & Fitted value & Unit \\
\hline$R R R$ & 175 & $\ldots$ \\
$\Delta x$ & 41 & $\mathrm{~nm}$ \\
$\Delta y$ & 1 & $\mathrm{~nm}$ \\
$\beta$ & 1 & $\cdots$ \\
$R_{\text {res } 1}$ & 25 & $\mathrm{n} \Omega$ \\
$R_{\text {res } 2}$ & 3 & $\mathrm{n} \Omega$ \\
$L$ & 1.6 & $\mathrm{pJ} / \mathrm{m}^{2}$ \\
\hline Derived parameter & Value & $\mathrm{Unit}$ \\
\hline$l=2.7 \cdot R R R$ & 472 & $\mathrm{~nm}$ \\
$\lambda$ & 39 & $\mathrm{~nm}$ \\
$\xi$ & 35 & $\mathrm{~nm}$ \\
$\lambda / \xi$ & 1.1 & $\cdots$ \\
$R_{\text {res }}(1 \mathrm{GHz})$ & 0.1 & $\mathrm{n} \Omega$ \\
$R_{s, Q \text {-inc }}(1 \mathrm{mT}, 1 \mathrm{GHz})$ & 10 & $\mathrm{n} \Omega$ \\
\hline \hline
\end{tabular}

of $l=475 \mathrm{~nm}$, a penetration depth $\lambda=39 \mathrm{~nm}$, and a coherence length $\xi=35 \mathrm{~nm}$. All these values come out as expected. The frequency dependent residual resistance $R_{\text {res }}$ ( $1 \mathrm{GHz}$ ) is $0.1 \mathrm{n} \Omega$, corresponding to a normal-conducting surface layer of $\Delta y=1 \mathrm{~nm}$ and less (Appendix).

As to the field-dependent surface resistance, two cases must be distinguished.

(i) The surface defects are normal conducting for all magnetic fields and densely packed. In that case they contribute to small Ohmic losses, independent of the rf frequency, because the supercurrent avoids them. They give rise to the frequency and magnetic field independent contribution to the surface resistance named $R_{\text {res } 2}=3 \mathrm{n} \Omega$.

(ii) The surface defects are more loosely connected and are superconducting at small magnetic fields, by proximity, but become normal conducting at larger magnetic fields. As the phase transition in the presence of a magnetic field is of first order, the defects absorb a constant amount of energy per transition and dissipate this energy per half rf cycle, causing the low field $Q$ increase. The contribution to the surface resistance is represented by $R_{s, Q \text {-inc }}(\omega)=10 \mathrm{n} \Omega$ at $f=1 \mathrm{GHz}$ and $B=1 \mathrm{mT}$. In addition their size increases with the magnetic field by penetration of magnetic flux, which is explained by a positive balance of condensation energy and magnetic energy in a type II superconductor such as niobium. Two cases must be distinguished. Either the phase transition occurs for a composite below the percolation threshold; then the contribution to the surface resistance is again purely Ohmic, but dependent on the magnetic field because of the size effect, represented by $R_{\text {res } 1}=25 \mathrm{n} \Omega$. Or the phase transition happens above the percolation threshold; then a supercurrent flows, growing with temperature and described by the percolation probability within a depth of $\Delta x=41 \mathrm{~nm}$, very close to the penetration depth $\lambda=39 \mathrm{~nm}$, as expected.

The inductive voltage of the supercurrent acts on the normal-conducting electrons, whose density increases by the action of the magnetic field. This effect causes the $Q$ slope at intermediate accelerating gradients. The losses originate from a mechanism very much the same as for the BCS surface resistance and show therefore similar frequency dependence. Close to $B_{c} / \kappa$ [cf. singularity in Eq. (38)], the rf losses increase beyond all limits, describing the $Q$ drop at larger accelerating gradients. The corresponding onset of the $Q$ drop depends critically on $\kappa$ and varies between 60 and $130 \mathrm{mT}$ (Fig. 6), which is close to the experimentally observed value, $80-110 \mathrm{mT}$ [52]. The corresponding contribution to the surface resistance is named $R_{s, f d}(B, T, \omega)$.

The percolation characteristics of the temperature dependent contribution to the surface resistance $R_{s, f d t}$ are illustrated in Fig. 4. The solid line follows the typical relation for the percolation transition as described by Eq. (46), with $\beta=1$, close to the two-dimensional percolation model prediction.

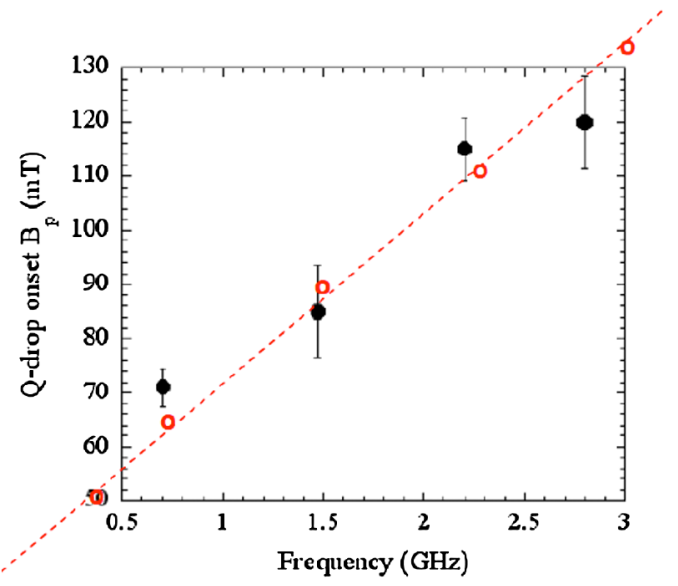

FIG. 6. $\quad Q$ drop onset field measured at different frequencies. The data point at $2.82 \mathrm{GHz}$ was measured in the $T E_{011}$ mode (from Ref. [52]). 
$Q\left[10^{9}\right]$

(a)

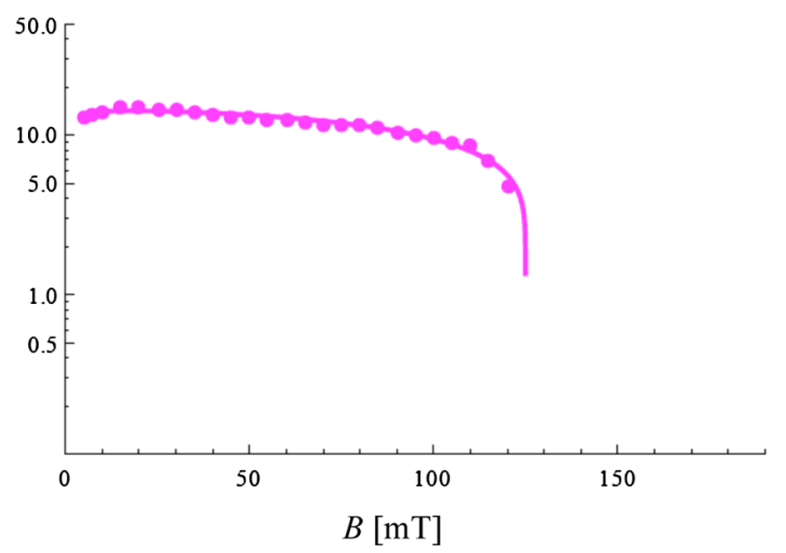

$\left.Q\left[10^{9}\right]\right]$

(b)

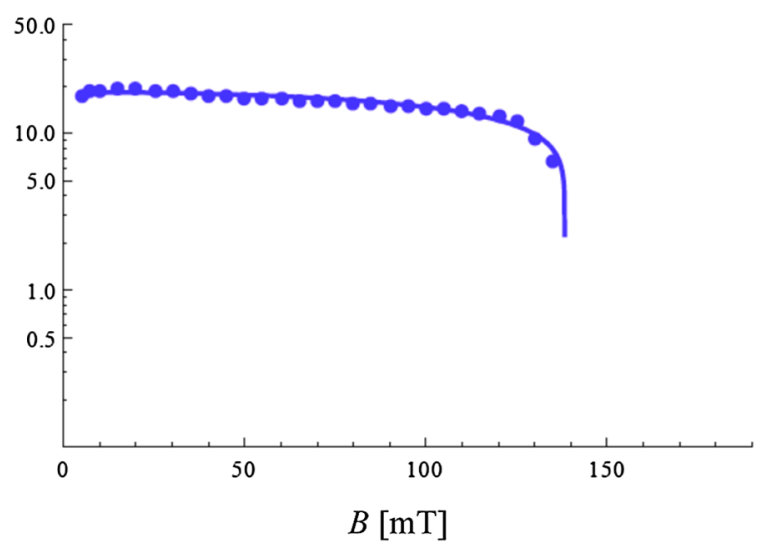

$\left.Q\left[10^{9}\right]\right]$

(c)

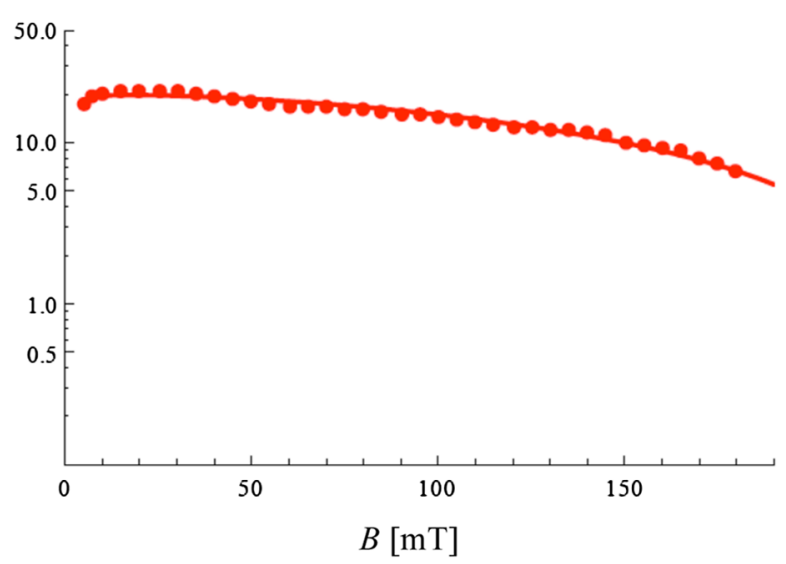

\begin{tabular}{|c|c|c|c|}
\hline & (a) & (b) & (c) \\
\hline & $\begin{array}{c}\text { Baseline } \\
\text { (EP) }\end{array}$ & $\begin{array}{c}\text { Heat } \\
\text { treated }\end{array}$ & $\begin{array}{c}120^{\circ} \mathrm{C} / 24 \mathrm{~h} \\
\text { bake }\end{array}$ \\
\hline \multicolumn{4}{|c|}{ Fitted parameters } \\
\hline$R R R$ & 266 & 199 & 179 \\
\hline$R_{\text {resl } 1}[\mathrm{n} \Omega]$ & 35 & 15 & 26 \\
\hline$L\left[\mathrm{pJ} / \mathrm{m}^{2}\right]$ & 6 & 1 & 5 \\
\hline$\kappa$ (surface) & 1.5 & 1.3 & 0.9 \\
\hline \multicolumn{4}{|c|}{ Fix or derivative parameters } \\
\hline$\beta$ & 1 & 1 & 1 \\
\hline$\lambda / \xi$ (bulk) & 1.1 & 1.1 & 1.1 \\
\hline$R_{\mathrm{Te} 22}[\mathrm{n} \Omega]$ & 1.5 & 1.5 & 1.5 \\
\hline$l[\mathrm{~nm}]$ & 719 & 538 & 482 \\
\hline$\Delta x[\mathrm{~nm}]$ & 0. & 0 . & 0. \\
\hline$\Delta y[\mathrm{~nm}]$ & 0. & 0 . & 0. \\
\hline
\end{tabular}

FIG. 7. Typical comparison of measured and fitted individual data: The $Q(B)$ curves were obtained for a $1300 \mathrm{MHz}$ single cell cavity at $2.0 \mathrm{~K}$ made of fine grain niobium. $\Delta x$ was intentionally set to 0 to avoid numerical instabilities during the fitting of data, because the $\Theta$ function already forces the respective term to 0 for $T<T^{*} . \Delta y$ was also intentionally set to 0 because it was small compared to the other numbers and otherwise oscillated between negative and positive values during the fitting of data.

The BCS part of the surface resistance $R_{s, \mathrm{BCS}}(T, \omega)$ is a property of the bulk and is independent of the presence of defects. The normal-conducting component of the charge carriers in the two-fluid model is exposed to the voltage of the superconducting component, resulting in a quadratic frequency dependence.

Hence, in total seven contributions to the surface resistance were identified, represented by the following symbols and dependencies on physical parameters: $R_{s, \mathrm{BCS}}(\omega, T), R_{\text {res }}(\omega), R_{\text {res } 1}, R_{\text {res } 2}, R_{s, f d t}(\omega, T), R_{s, f d b}(B)$, and $R_{s, Q \text {-inc }}(\omega, B)$. The combination of $R_{\text {res } 1}, R_{s, f d t}(\omega, T)$, $R_{s, f d b}(B)$ is responsible for the $Q$ slope and $Q$ drop: $R_{s, f d}(\omega, T, B)=\left[R_{\text {res } 1}+R_{s, f d t}(\omega, T)\right] \cdot R_{s, f d b}(B)$.

\section{Typical fit results on individual data}

It is good practice to compare the results of the present analysis with other experimental findings. Reference data are taken from a thorough analysis on the $Q$ slope and $Q$

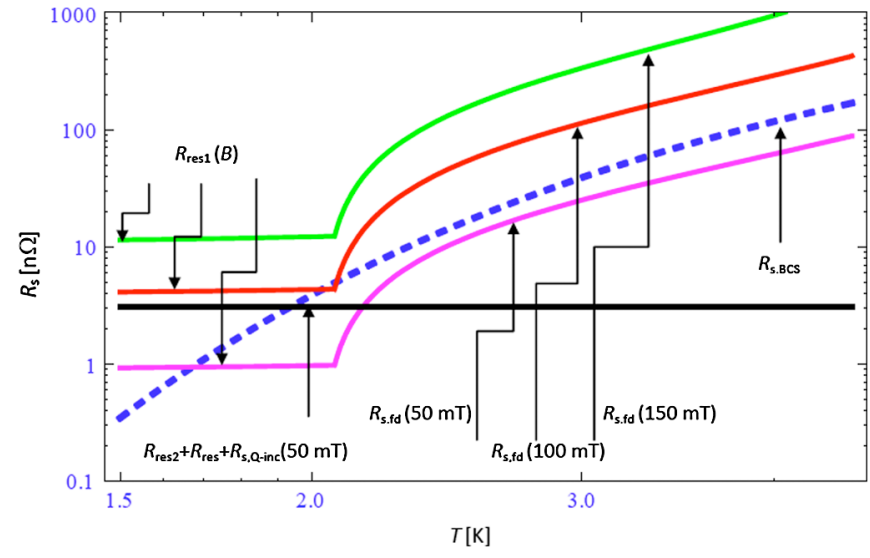

FIG. 8. Contributions to the surface resistance at $704 \mathrm{MHz}$ shown separately (green: $R_{s, f d}$ at $150 \mathrm{mT}$; red: $R_{s, f d}$ at $100 \mathrm{mT}$; magenta: $R_{s, f d}$ at $50 \mathrm{mT}$; dashed blue: $R_{s, \mathrm{BCS}}$; black: sum of residual resistances $R_{\text {res }}$ and $R_{\text {res 2). }}$. 
drop performed several years ago $[53,54]$. These data suggest also a linear dependence of the surface resistance $R_{S}$ on the magnetic field $B$, in addition to the quadratic and higher dependencies as outlined in this study and elsewhere (a summary of models proposed can be found in Ref. [52]). The present study provides no evidence for a linear dependence, though, in accordance to Ref. [1].

Other data from Ref. [52] concern the onset field for the $Q$ drop, measured at different frequencies (Fig. 6). The $Q$ drop onset field is taken from the fit to the collective data at a $Q$ value of about $75 \%$ of its maximum (open circles and dotted line) and represents well the experimental data of Fig. 6.

Figure 7 shows typical results of individual fits to selected data sets taken out of the collective data sample. The three curves were obtained from data [13] on the same cavity that had been subjected to the sequence of treatments indicated, all other parameters kept unchanged.

The results of the fit allow an explanation of the beneficial effect of the low temperature bake, which was found to eliminate the $Q$ drop. It can be seen that mainly two parameters are affected by the bakeout. The first is the Ginzburg-Landau parameter $\kappa$ [Eq. (39)], which is significantly reduced with the treatment number. As $\kappa$ determines the entry of magnetic flux at the surface it must be taken as a physical property of the surface. The other parameter is the $R R R$, which decreases from one treatment to the next. It is a physical property of the bulk, but restricted to the penetration depth. All other parameters are nearly unaffected. This observation can be explained by a redistribution of impurities from the very surface into the bulk up to the penetration depth. This interpretation is in line with current knowledge attributing the elimination of the $Q$ drop to a critical interplay of oxygen diffusion into the bulk and decomposition of the oxide at the surface [55].

\section{Contributions to the surface resistance at $704 \mathrm{MHz}$}

It is important to know the relative importance of the different contributions to the surface resistance of accelerating cavities, as, for example, planned for proton drivers with a typical frequency of $704 \mathrm{MHz}$ [56]. It is clearly visible in Fig. 8 how the total surface resistance remains nearly constant below and increases beyond the threshold temperature of $2 \mathrm{~K}$.

\section{CONCLUSION}

A quantitative relation for the total surface resistance $R_{s}$ is established including the $Q$-slope/ $Q$-drop/low field $Q$ increase, as observed in superconducting bulk niobium cavities. The proposed physical model allows the description of all three phenomena altogether. The $Q$-slope/ $Q$-drop is explained by the gradual entry of magnetic flux with increasing magnetic field amplitude at surface defects that act as nucleation centers and a rapid entry of magnetic flux close to $B_{c} / \kappa$. The low field $Q$ increase is explained by the latent heat dissipated when weak superconducting defects undergo a phase transition to the normal and back to the superconducting state. The rf losses are explained by the conjecture that along with the entry of magnetic flux the density of the normal-conducting electrons increases as well. These electrons feel the inductive voltage of the superconducting electrons, thus raising the rf losses. Percolation and proximity effects explain in detail the experimental observations of a large sample of collective cavity test data. A fitting procedure with a relatively small number of free parameters (Fig. 9) allows a valid representation of the experimental $Q(B)$ curves of the individual cavity tests and also the determination of the model-relevant physical parameters, which are in reasonable agreement with present knowledge.

\section{ACKNOWLEDGMENTS}

This work was performed during my longstanding membership in the rf group of CERN's AB and BE departments. It would not have been possible without the support and the freedom that, beyond the daily tasks of the group, was provided to me. I would like to express my thanks to T. Linnecar and E. Ciapala, in charge of the rf group, to O. Brunner, who manages the activities of superconducting cavities, as well as to T. Junginger and J. Tückmantel for many fruitful discussions.

\section{APPENDIX: LEAST SQUARE FIT RESULTS ON THE COLLECTIVE DATA}

$$
\begin{aligned}
R_{s, \mathrm{BCS}}(\omega, T) & =\mu_{0}^{2} \omega^{2} \sigma_{n 0} \lambda^{3} \frac{\Delta}{k_{B}} \ln \left(\frac{\Delta}{\hbar \omega}\right) \frac{e^{-\Delta / k_{B} T}}{T} ; \quad \sigma_{n 0}=R R R \sigma ; \quad \lambda(T, l)=\frac{\lambda_{0}(l)}{\sqrt{1-\left(\frac{T}{T_{c}}\right)^{4}}} ; \quad \lambda_{0}(l)=\lambda_{L} \sqrt{1+\frac{\xi_{0}}{l}} \\
l l_{\mathrm{nm}} & =2.7 R R R ; \quad R_{s, f d}(\omega, T, B) \approx[R_{\mathrm{res} 1}+\underbrace{\mu_{0}^{2} \omega^{2} \Delta x^{3} \sigma_{n 0} \Theta\left(T-T^{*}\right)\left(\frac{T-T^{*}}{T_{c}-T^{*}}\right)^{\beta}}_{R_{s, f d t}}] \underbrace{\frac{1}{\kappa^{2}\left(\frac{\left(\frac{\kappa B}{B_{c}}\right)^{2}}{2}+\frac{\left(\frac{\kappa B}{B_{c}(T)}\right)^{4}}{3}+\cdots\right)}}_{R_{s, f d b}} \\
R_{\mathrm{res}}(\omega) & =\mu_{0}^{2} \sigma_{n 0} \omega^{2} \lambda^{2} \Delta y ; \quad R_{s, Q \text {-inc }} \approx \frac{2 \omega L \mu_{0}^{2}}{\pi B^{2}} ; \quad R_{\text {res } 2}=\text { const. }
\end{aligned}
$$



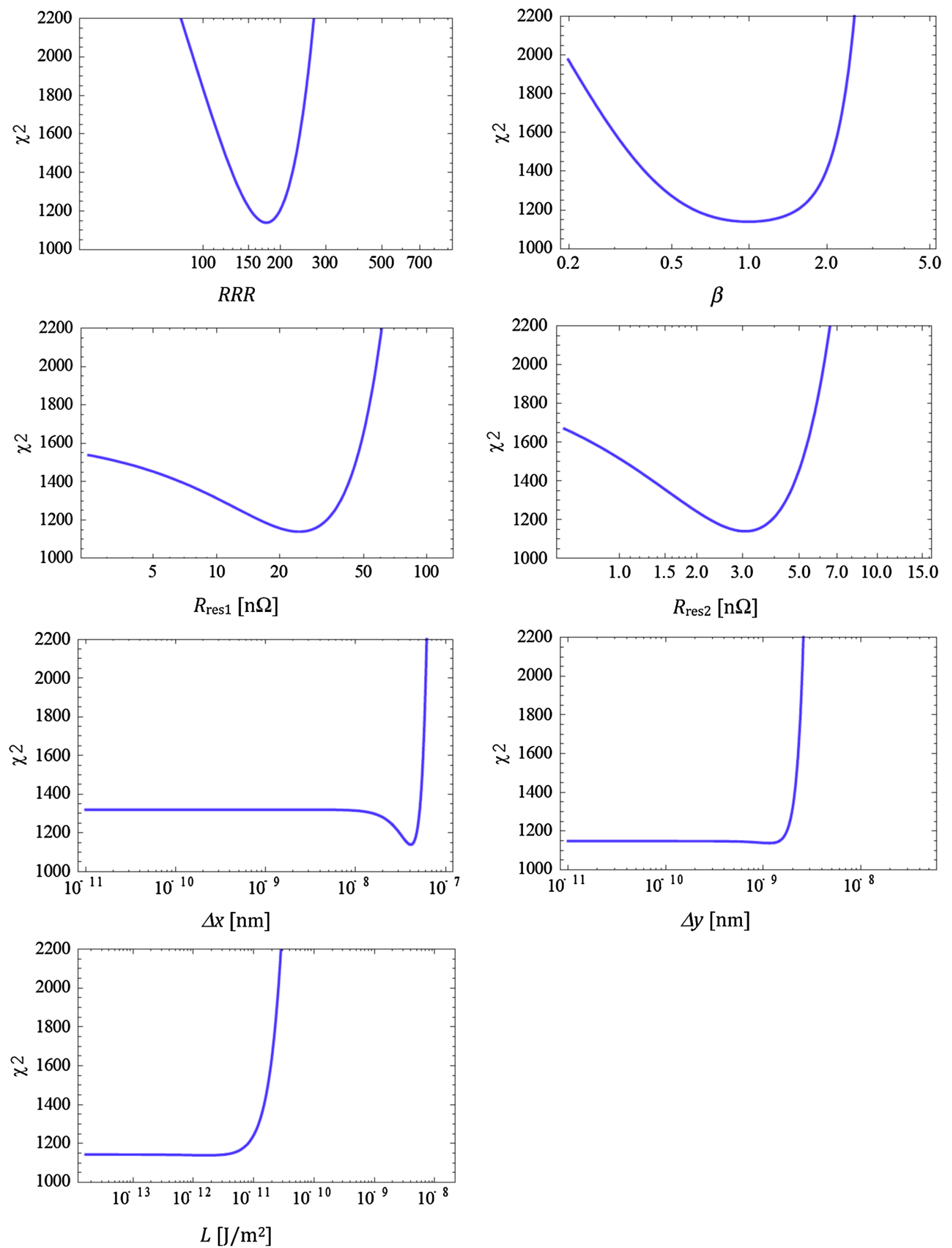

FIG. 9. Results of the $\chi^{2}$ minimization of the fit parameters related to the surface resistance $R_{s}$. 
[1] B. Visentin, in Proceedings of the Workshop "Pushing the Limits of RF Superconductivity," 2004, edited by KwangJe Kim and Catherine Eyberger, 2005 (Argonne National Laboratory Report No. ANL-05/10, p. 299); ICFA Beam Dynamics Newsletter 39, 94 (2006).

[2] K. Halbach and R.F. Holsinger, Part. Accel. 7, 213 (1976).

[3] K. Akai et al., Nucl. Instrum. Methods Phys. Res., Sect. A 499, 45 (2003).

[4] N. Akaoka et al., in Proceedings of the 9th International Workshop RF Superconductivity (SRF1999) (Los Alamos National Laboratory, Santa Fe, NM, 2000).

[5] C. Arnaud et al., in Proceedings of the 14th International Conference on High-Energy Accelerators, Tsukuba, Japan, 1989 [Part. Accel. 25-33, 749 (1990)]; Proceedings of the 4th Workshop RF Superconductivity (KEK, Tsukuba, Japan, 1989).

[6] G. Arnolds-Mayer et al., IEEE Trans. Nucl. Sci. 32, 3587 (1985).

[7] P. Bauer et al., Physica (Amsterdam) 441C, 51 (2006) [http://www.lepp.cornell.edu/public/SRF2005/].

[8] Ph. Bernard et al., Report No. CERN/EF/RF-85-6, 1985.

[9] J.L. Biarrotte et al., Proceedings of the 9th International Workshop RF Superconducting (SRF1999) Santa Fe, NM (Ref. [4]).

[10] S. Bousson, in Proceedings of the 10th Workshop RF Superconductivity (KEK, Tsukuba, Japan, 2001).

[11] K. C. D. Chan et al., Proceedings of the 9th International Workshop RF Superconducting (SRF1999) Santa Fe, NM (Ref. [4]).

[12] G. Ciovati, J. Appl. Phys. 96, 1591 (2004).

[13] G. Ciovati, in 14th International Conference on $R F$ Superconductivity (SRF2009) (Helmholtz Zentrum Berlin, Berlin, Germany, 2009).

[14] D. Dasbach et al., IEEE Trans. Magn. 25, 1862 (1989).

[15] G. Devanz, HIPPI Annual Meeting, CERN, 2008.

[16] H. Diepers et al., Phys. Lett. 38A, 337 (1972).

[17] F. Furuta et al., in Proceedings of LINAC 2006 (Oak Ridge National Laboratory, Knoxville, Tennessee, 2006), TUP025.

[18] H. Hayano et al., TTC Meeting, DESY, 2008; T. Kasuga et al., in Proceedings of the 2007 Particle Accelerator Conference, Albuquerque, New Mexico (IEEE, New York, 2007).

[19] K. Hosoyama et al., in Proceedings of the 12th International Workshop RF Superconducting (SRF2005), Ithaca, NY [http://www.lepp.cornell.edu/public/SRF2005/].

[20] Y. Kojima et al., in Proceedings of the 1989 Workshop RF Superconducting, Tsukuba, Japan (Ref. [5]).

[21] L. Lilje et al., Nucl. Instrum. Methods Phys. Res., Sect. A 524, 1 (2004); L. Lilje et al., XFEL Design Report, DESY, Hamburg, Germany.

[22] H. Nakai et al., in Proceedings of the 10th Workshop RF Superconducting, Tsukuba, Japan, 2001 (Ref. [10]).

[23] N. Ouchi et al., IEEE Trans. Appl. Supercond. 9, 1030 (1999).

[24] C. Pagani, in Proceedings of the 10th Workshop RF Superconducting, Tsukuba, Japan, 2001 (Ref. [10]).

[25] J. Plouin, HIPPI Annual Meeting, CERN 2008.

[26] C. Reece, in Proceedings of the 10th Workshop RF Superconducting, Tsukuba, Japan, 2001 (Ref. [10]).
[27] C. H. Rode, IEEE Trans. Appl. Supercond. 9, 873 (1999).

[28] Y. Yamamoto, TESLA Technology Collaboration (TTC) Meeting, Milano, Italy, 2011.

[29] R. York et al., Project X Workshop 2008, Fermilab, Batavia, IL; W. Hartung et al., in Proceedings of LINAC 2006, Knoxville, Tennessee (Ref. [17]).

[30] H. Frey and R.H. Haefer, Tieftemperaturtechnolgie (VDI-Verlag, Düsseldorf, 1981).

[31] J. Amrit and M. X. Francois, J. Low Temp. Phys. 119, 27 (2000).

[32] A. W. Chao and M. Tigner, Handbook of Accelerator Physics (World Scientific, Singapore, 1999).

[33] C. J. Gorter and H.B.G. Casimir, Z. Phys. 15, 539 (1934); Phys. Z. 35, 963 (1934)

[34] D. C. Mattis and J. Bardeen, Phys. Rev. 111, 412 (1958).

[35] H. Padamsee, RF Superconductivity, Science, Technology, and Applications (Wiley-VCH, Weinheim, Germany, 2009), p. 42, and references therein.

[36] P. Kneisel, O. Stoltz, and J. Halbritter, in Proceedings of the Particle Accelerator Conference, Chicago, IL, 1971 (IEEE, New York, 1971); IEEE Trans. Nucl. Sci. 18, 158 (1971).

[37] H. J. Halama, Part. Accel. 2, 335 (1971).

[38] G. Arnolds-Mayer and W. Weingarten, Report No. CERN/EF 86-20, 1986; IEEE Trans. Magn. 23, 1620 (1987).

[39] C. Benvenuti, S. Calatroni, I. E. Campisi, P. Darriulat, M. A. Peck, R. Russo, and A.-M. Valente, Physica (Amsterdam) 316C, 153 (1999).

[40] M. Grundner and J. Halbritter, J. Appl. Phys. 51, 397 (1980).

[41] C. Antoine and S. Berry, in Proceedings of the First International Workshop on Hydrogen in Materials and Vacuum Systems, AIP Conference Proceedings No. 671 (AIP, Melville, NY, 2003), p. 176.

[42] G. Deutscher and P. G. de Gennes, in Superconductivity, edited by R. Parks (M. Dekker, New York, 1969), p. 1005.

[43] P. G. de Gennes and J.P. Hurault, Phys. Lett. 17, 181 (1965).

[44] N. W. Ashcroft and N.D. Mermin, Solid State Physics (Holt, Rinehart and Winston, New York, 1976).

[45] E. R. Pollard, Ph.D. thesis, MIT, Cambridge, MA, 1968.

[46] L. N. Cooper, Phys. Rev. Lett. 6, 689 (1961).

[47] M. D. Rintoul, Phys. Rev. E 62, 68 (2000).

[48] A. Bunde and W. Dieterich, J. Electroceram. 5, 81 (2000).

[49] J. P. Straley, Phys. Rev. B 15, 5733 (1977).

[50] J. Kotzler, L. von Sawilski, and S. Casalbuoni, Phys. Rev. Lett. 92, 067005 (2004).

[51] U. Klein, Ph.D. thesis, Wuppertal University, 1981; G. Müller, Ph.D. thesis, Wuppertal University, 1983; J.P. Turneaure, J. Halbritter, and H. A. Schwettman, J. Supercond. 4, 341 (1991).

[52] G. Ciovati, J. Appl. Phys. 96, 1591 (2004).

[53] G. Ciovati and J. Halbritter, Ref. [7], p. 57.

[54] G. Ciovati, Ref. [7], p. 44.

[55] G. Ciovati, Appl. Phys. Lett. 89, 022507 (2006).

[56] O. Brunner, S. Calatroni, E. Ciapala, M. Eshraqi, R. Garoby, F. Gerigk, A. Lombardi, R. Losito, V. Parma, C. Rossi, J. Tuckmantel, M. Vretenar, U. Wagner, and W. Weingarten, Phys. Rev. ST Accel. Beams 12, 070402 (2009). 